\title{
Pharmacokinetics for Once- Versus Twice-Daily Tacrolimus Formulations in De Novo Kidney Transplantation: A Randomized, Open-Label Trial
}

\author{
Z. Wlodarczyk ${ }^{a}$, J.-P. Squifflet ${ }^{b}$, M. Ostrowskic, \\ P. Rigotti ${ }^{d}$, S. Stefoni ${ }^{\mathrm{e}}$, F. Citterio ${ }^{f}$, \\ Y. Vanrenterghem ${ }^{g}$, B. K. Krämerh, \\ D. Abramowicz' , F. Oppenheimeri, \\ F. Pietruck' ${ }^{k}$, G. Russ', C. Karpf ${ }^{m}$ and N. Undren,* \\ a Szpital Uniwersytecki, Bydgoszcz, Poland \\ ' University of Liege, CHU Sart Tilman, Liege, Belgium \\ (formerly of Cliniques Universitaires Saint-Luc, Brussels, \\ Belgium) \\ ${ }^{c}$ Klinika Chirurgii Ogolnej I Transplatacyjnej, Szczecin, \\ Poland \\ ${ }^{\mathrm{d}}$ Azienda Ospedaliera di Padova, Padova, Italy \\ e Ospedale Policlinico S. Orsola, Bologna, Italy \\ ${ }^{\dagger}$ Policlinico Universitario Agostino Gemelli, Rome, Italy \\ g Universitaires Ziekenhuizen Gasthuisberg, Leuven, \\ Belgium \\ ${ }^{\mathrm{h}}$ Marienhospital Herne, Klinikum der Ruhr-Universität \\ Bochum, Herne, Germany (formerly of Klinikum der \\ Universität Regensburg, Regensburg, Germany) \\ 'Clinique Universitaires Hospital Erasme, Brussels, \\ Belgium \\ jHospital Clinic i Provincial, Barcelona, Spain \\ kUniversity of Duisburg-Essen, Essen, Germany \\ 'Queen Elizabeth Hospital, Adelaide, Australia \\ m Astellas GmbH, Munich, Germany (formerly Fujisawa, \\ Germany at time of study) \\ ${ }^{n}$ Astellas Pharma Europe Limited, Staines, UK (formerly \\ Fujisawa, Germany at time of study) \\ * Corresponding author: Nasrullah Undre, \\ Nas.undre@eu.astellas.com
}

Tacrolimus, a cornerstone immunosuppressant, is widely available as a twice-daily formulation (Tacrolimus BID). A once-daily prolonged-release formulation (Tacrolimus QD) has been developed that may improve adherence and impart long-lasting graft protection. This study compared the pharmacokinetics (PK) of tacrolimus in de novo kidney transplant patients treated with Tacrolimus QD or Tacrolimus BID. A 6-week, open-label, randomized comparative study was conducted in centers in Europe and Australia. Eligible patients received Tacrolimus QD or Tacrolimus BID. PK profiles were obtained following the first tacrolimus dose (day 1), and twice under steady-state conditions. As secondary objectives, efficacy and safety parameters were also evaluated. Sixty-six patients completed all PK profiles (34 Tacrolimus QD, 32 Tacrolimus BID). Mean AUC $\mathrm{C}_{0-24}$ of tacrolimus on day 1 was approximately $30 \%$ lower for Tacrolimus QD than Tacrolimus BID (232 and $361 \mathrm{ng} . \mathrm{h} / \mathrm{mL}$, respectively), but was comparable by day 4 . There was a good correlation and a similar relationship between $\mathrm{AUC}_{0-24}$ and $\mathrm{C}_{\min }$ for both formulations. Efficacy and safety data were also comparable over the 6-week period. Tacrolimus $Q D$ can be administered once daily in the morning on the basis of the same systemic exposure and therapeutic drug monitoring concept as Tacrolimus BID.

Key words: Kidney transplantation, once-daily tacrolimus, pharmacokinetics, prolonged-release tacrolimus, tacrolimus

Received 26 February 2009, revised 16 June 2009 and accepted for publication 29 June 2009

\section{Introduction}

Nonadherence, also referred to as noncompliance, is one of the risk factors for poor long-term outcomes following solid-organ transplantation, and is a leading cause of preventable graft loss $(1,2)$. As such, interventions that improve adherence to immunosuppressive therapy should significantly improve graft survival after renal transplantation (3). A significant association has been demonstrated between frequency of dosing regimen and likelihood of nonadherence to immunosuppressants in transplant patients $(4,5)$. Also, in renal transplant patients, morning dosing is associated with significantly higher adherence than evening dosing (5). These findings suggest that a oncedaily (OD) morning dosing regimen maximizes the potential for adherence and may, consequently, improve long-term outcomes.

Tacrolimus (Prograf ${ }^{\circledR}$; Astellas Pharma Europe, Staines, UK) is an established immunosuppressant used for the prevention and treatment of allograft rejection in kidney, liver and heart transplantation; in clinical practice, this formulation is usually administered twice daily (BID), and hereafter in this article will be referred to as Tacrolimus BID. QD tacrolimus (Advagraf ${ }^{\circledR}$; Astellas Pharma Europe, Staines, UK) is a prolonged-release formulation that was developed to enable a OD dosing alternative and to have similar safety and efficacy profiles to Tacrolimus BID with the potential 


\section{Wlodarczyk et al.}

to improve patient adherence and quality of life; hereafter this formulation will be referred to as Tacrolimus QD.

Tacrolimus has a narrow therapeutic index $(6,7)$ and its oral bioavailability is highly variable between individuals (8). Systemic exposure to tacrolimus (area under the curve, AUC) is a significant efficacy variable and, therefore, therapy is optimized on an individual patient basis by monitoring trough levels as surrogate markers of exposure. Thus, in developing Tacrolimus QD, the aim was to use the same therapeutic monitoring concepts as for Tacrolimus BID.

Tacrolimus pharmacokinetics (PK) have been compared between Tacrolimus QD and Tacrolimus BID in stable kidney, liver and heart transplant patients (9-11). However, the PK of Tacrolimus QD in newly transplanted kidney recipients has not been previously investigated. The objective of this study was to compare the PK parameters of tacrolimus following first administration and under steady-state conditions in de novo kidney transplant recipients treated with Tacrolimus QD or Tacrolimus BID.

\section{Methods}

\section{Study design and procedures}

This was a 6-week, phase II, multicenter, open, prospective, 1:1 randomized, comparative PK study of tacrolimus administered as Tacrolimus QD or Tacrolimus BID in patients undergoing kidney transplantation. Patients were allocated to treatment groups, locally at each center, using sealed randomization envelopes. Randomization was performed preoperatively on a $1: 1$ (Tacrolimus QD : Tacrolimus BID) basis stratified by center, with each center being allocated a unique sequence of patient numbers. Patients were either being treated in hospital or attended clinic at the time of randomization (baseline; day 0 ) and on days 1, 3, 7, 11, 14, week 4 and week 6 ( \pm 7 days). As this was an open-label study, no blinding was necessary. At the end of the study period, patients in the Tacrolimus QD group had the option to continue treatment with Tacrolimus QD as part of a long-term extension study. One-year follow-up results are also reported here.

\section{Patients}

Patients with end-stage renal disease were eligible for enrollment if they were 18-65 years of age, suitable candidates for renal transplantation, receiving a kidney transplant from a deceased or living donor between 5 and 65 years of age with a compatible ABO blood type, and had provided written informed consent prior to enrollment.

Exclusion criteria included a panel reactive antibody grade $>50 \%$ in the previous 6 months and/or previous graft survival $<1$ year for immunological reasons; kidney graft donation after cardiac death; cold ischemia time of the donor kidney $>40 \mathrm{~h}$; systemic infection requiring treatment; significant liver disease; previous organ transplantation other than kidney; contraindication for the use of tacrolimus; severe diarrhea, vomiting, an active peptic ulcer or a gastrointestinal disorder that may have affected the absorption of tacrolimus; history of diabetes mellitus; malignancy or history of malignancy within the previous 5 years (except successfully treated nonmetastatic basal or squamous cell carcinoma of the skin); and likelihood of nonadherence to the study protocol. Patients and donors were also excluded if they were known to be positive for hepatitis B virus, hepatitis $C$ virus or human immunodeficiency virus.
To enter into the extension phase of this study, patients must have been fully informed and must have given written informed consent to demonstrate comprehension of the purpose and risks of the study. Patients were excluded from the follow-up phase if they were pregnant, nursing or not using adequate contraception during the study.

Patients were enrolled into centers in Australia, Belgium, France, Germany, Italy, Poland, Spain and Switzerland. The study was conducted in accordance with the Declaration of Helsinki. The protocol was reviewed by the Ethics Committee at each study center and each patient gave written informed consent prior to enrollment into the study.

\section{Study treatment}

Following transplantation, patients were randomized to receive either Tacrolimus QD or Tacrolimus BID in combination with mycophenolate mofetil (MMF) and corticosteroids. The first dose of tacrolimus was administered within $24 \mathrm{~h}$ of reperfusion on the morning following transplantation; Tacrolimus QD was administered as a single dose and Tacrolimus BID as two divided doses. Subsequent doses of Tacrolimus QD (once daily, in the morning) and Tacrolimus BID (twice daily) were taken orally on an empty stomach or at least $1 \mathrm{~h}$ before or 2-3 $\mathrm{h}$ after a meal. The initial dose of tacrolimus was $0.2 \mathrm{mg} / \mathrm{kg} / \mathrm{day}$. Subsequent doses were adjusted to maintain tacrolimus trough levels in the range of $10-20 \mathrm{ng} / \mathrm{mL}$ (days 1-14) and $5-15 \mathrm{ng} / \mathrm{mL}$ (day 15 to week 6).

A standardized regimen of MMF was used, with a starting dose of $2 \mathrm{~g} /$ day, reduced to $1 \mathrm{~g} /$ day after 14 days in clinically stable patients. An intravenous (IV) bolus (500-1000 mg) of methylprednisolone (or equivalent) was administered prior to reperfusion. A second IV dose of 125-250 mg was administered in conjunction with the first dose of tacrolimus (Tacrolimus $\mathrm{QD}$ or Tacrolimus BID). Oral prednisone (or equivalent) was also permitted at a dose of 15-20 mg/day during the first month and in tapered doses thereafter.

\section{Pharmacokinetic profiles and assay}

Three 24-h blood concentration-time profiles were taken during the study. Each profile consisted of 17 blood samples that were collected predose and at $0.5,1,2,3,4,6,8,12,12.5,13,14,15,16,18,20$ and $24 \mathrm{~h}$ after dosing (Figure 1). Assays were based on whole blood ( $2 \mathrm{~mL}$ aliquots) in ethylene diamine tetraacetic acid tubes. Blood samples were frozen at $-20{ }^{\circ} \mathrm{C}\left(-4{ }^{\circ} \mathrm{F}\right)$ within $2 \mathrm{~h}$ of collection and stored until shipment to a central laboratory (Covance Laboratories Ltd, Harrogate, UK) for analysis. Tacrolimus concentrations were determined, using validated high-performance liquid chromatography tandem mass spectroscopy (HPLC/MS/MS) assay methods (lower limit of quantification $0.1 \mathrm{ng} / \mathrm{mL}$ ). The assay was based on the method developed by Alak et al. (12). Wholeblood calibration standards, quality control samples and study samples were thawed and $1-\mathrm{mL}$ aliquots were taken. Internal standard $(20 \mu \mathrm{L}, 50 \mathrm{ng} / \mathrm{mL}$ ) was added and mixed briefly. Aliquots were extracted using protein precipitation and solid-phase extraction using C18 200-mg/3-mL extraction cartridges. Elutes were evaporated to dryness under a stream of nitrogen at $40^{\circ} \mathrm{C}$, and residues were redissolved in a $50: 50 \mathrm{mix}(\mathrm{v} / \mathrm{v})$ of acetonitrile and water, mixed and centrifuged, before being submitted for HPLC/MS/MS.

For routine monitoring, microparticle enzyme immunoassay $\left(\mathrm{IMx}{ }^{\circledR}\right)$ or enzyme multiplied immunoassay technique were used. As an additional aid for dose adjustments, limited sampling AUC monitoring was performed on days $1,3,7,11$ and 14 and as clinically indicated. Blood samples were collected at 0 (predose) and at 2, 4, 8 and 12-h postdose for both formulations, with an additional sample at 24-h postdose for Tacrolimus QD, and assayed at the investigational site. The resulting data were sent to an external panel (Covance Clinical Research Unit Ltd., Leeds, UK) to estimate AUC using the standard linear trapezoidal method. 


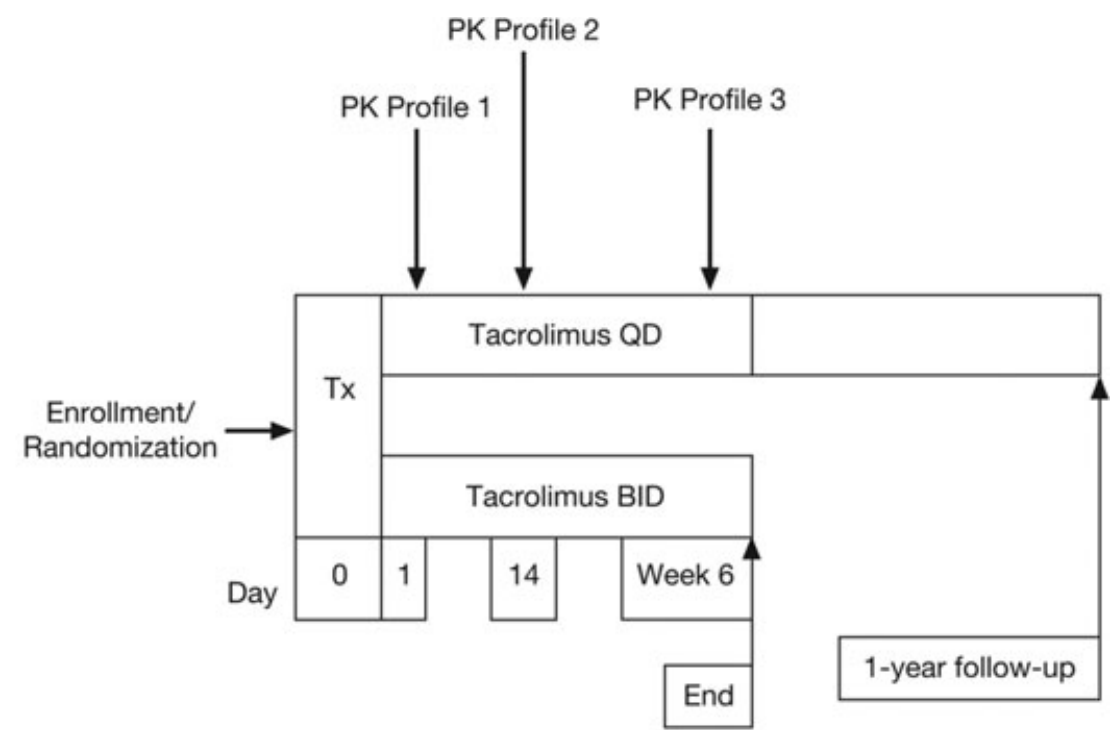

Figure 1: Study design and schedule of PK profiles.

\section{4 hour blood concentration-time profile}

\begin{tabular}{|l|l|l|l|l|l|l|l|l|l|l|l|l|l|l|l|l|l|l|l|}
\hline Hour & 0 & 0.5 & 1 & 2 & 3 & 4 & 6 & 8 & 12 & 12.5 & 13 & 14 & 15 & 16 & 18 & 20 & 24 \\
\hline
\end{tabular}

The AUC values were immediately made available to the investigational site to aid estimation of exposure; the decision to make any dose adjustments was the responsibility of the site investigator. All procedures were performed in compliance with the principles of Good Laboratory Practice.

\section{Objectives and endpoints}

The objective of this study was to compare the PK parameters of tacrolimus following first administration and under steady-state conditions in de novo kidney transplant recipients treated with Tacrolimus QD or Tacrolimus BID.
The primary endpoint was a comparison of systemic exposure $\left(\mathrm{AUC}_{0}-24\right)$ between Tacrolimus QD and Tacrolimus BID on day 1, day 14 and week 6 after transplantation in the PK Evaluable Set (all patients who received all doses of study medication, provided three evaluable PK profiles and had no major PK protocol deviations). Secondary endpoints included:
Figure 2: Patient disposition. Reasons for withdrawal from PK evaluable set classified as 'other', Tacrolimus QD: failures to complete a PK profile $(\mathrm{n}=$ 5), medication or dosing errors ( $\mathrm{n}=$ $3)$, diagnosis of breast cancer $(n=1)$; Tacrolimus BID: protocol violations (diabetes, hemodialysis) ( $n=2)$, failures to complete a PK profile $(n=2)$, medication errors $(n=2)$, treatment for rejection $(n=1)$, graft failure $(n=1)$.

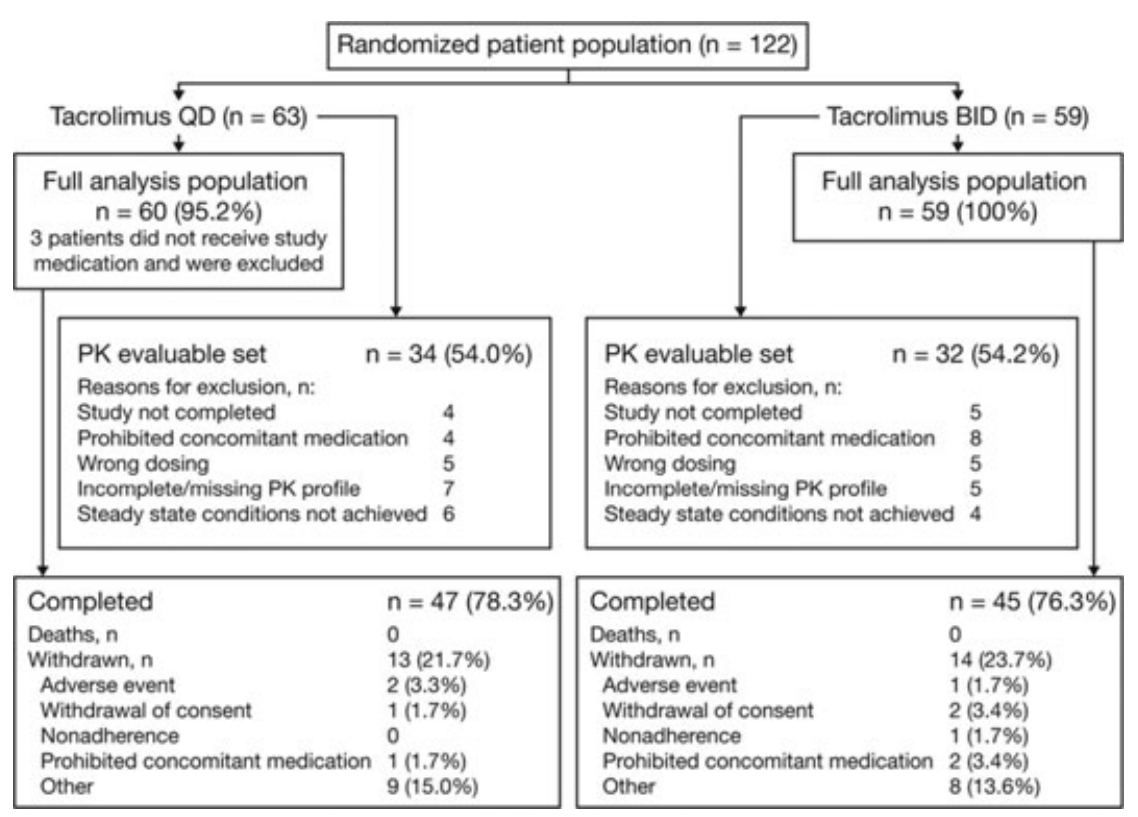


- correlation between mean $\mathrm{C}_{\text {min }}$ and mean AUC (PK Evaluable Set);

- incidence and severity of, and time to, biopsy-proven acute rejection (BPAR) episodes during the 6-week study;

- 6-week patient and graft survival in the Full Analysis Set (all patients randomized into the study who received at least one dose of study medication);

- incidence of adverse events (AEs) at 6 weeks (Full Analysis Set);

- laboratory measurements for hematology and biochemistry (performed at baseline, at the time of PK profiling and at week 4).

\section{Statistical analyses}

Assuming a steady-state $\mathrm{AUC}_{0-24}$ ratio of 0.9 and a standard deviation (SD) of 0.35 for each treatment arm, it was estimated that 32 patients with three complete PK profiles would have a power of nearly $80 \%$ to assess equivalence in exposure. Taking into consideration potential dropout rates, it was planned to enroll approximately 40 patients per treatment arm. PK parameters for Tacrolimus QD and Tacrolimus BID (AUC $\mathrm{C}_{0-24}, \mathrm{C}_{\max }$ and $\mathrm{C}_{24}$ ) were evaluated using a two-sided $90 \%$ confidence interval for the ratio of means, with an acceptance interval of $80-125 \%$. All PK parameters were calculated from the complete 24 -h concentration-time profiles of tacrolimus in whole blood resulting from administration of Tacrolimus QD or Tacrolimus BID. AUC was estimated using the linear trapezoidal rule. $\mathrm{C}_{\max }$, $\mathrm{T}_{\max }$ and $\mathrm{C}_{12}$ or $\mathrm{C}_{24}$ were obtained directly from the concentration-time profiles.

\section{Results}

\section{Study population}

A total of 122 patients were enrolled into the study. More patients than originally planned were recruited due to actual dropout rates. Recruitment was continued until both treatment groups had a minimum of 32 patients with three complete PK profiles (Figure 2).

Three patients did not receive study medication, thus the Full Analysis Set comprised 119 patients (60 Tacrolimus QD; 59 Tacrolimus BID). Of 92 patients who completed the 6-week treatment period, 66 patients (34 Tacrolimus QD; 32 Tacrolimus BID) met the criteria for inclusion in the PK Evaluable Set. There were no deaths during the study and patient disposition was similar between the two treatment groups (Figure 2).

Data were collected between March 2003 and March 2004. The first patient was randomized on March 6, 2003, and the last patient visit was on March 2, 2004. Demographic and baseline characteristics of patients and donors, for both the Full Analysis Set and the PK Evaluable Set, were comparable between the two treatment groups. A majority of patients were male Caucasians (Table 1).

\section{Drug administration and exposure}

The mean total daily doses of both Tacrolimus QD and Tacrolimus BID remained stable throughout the study (Figure 3A). Apart from week 1, the mean Tacrolimus OD dose was higher than the corresponding Tacrolimus BID dose (but was not significantly different). Trough levels
Table 1: Baseline demographics and disease characteristics

\begin{tabular}{|c|c|c|}
\hline Full Analysis Set & $\begin{array}{l}\text { Tacrolimus QD } \\
(n=60) \\
\text { Patients (\%) }\end{array}$ & $\begin{array}{c}\text { Tacrolimus } \\
\text { BID ( } n=59) \\
\text { Patients (\%) }\end{array}$ \\
\hline Male & $34(56.7)$ & $44(74.6)^{2}$ \\
\hline Female & 26 (43.3) & $15(25.4)$ \\
\hline Age (years) $^{1}$ & $44.0(19-66)$ & $43.6(21-65)$ \\
\hline Height $(\mathrm{cm})^{1}$ & 169.3 (149-193) & $168.9(148-184)$ \\
\hline Weight $(\mathrm{kg})^{1}$ & $70.2(41-115)$ & $69.3(40-100)$ \\
\hline Caucasian & $58(96.7)$ & 59 (100) \\
\hline Black & 0 & 0 \\
\hline Asian & 0 & 0 \\
\hline Other & $2(3.3)$ & 0 \\
\hline $\begin{array}{l}\text { Existing glucose } \\
\text { metabolism disorder }\end{array}$ & $1(1.7)$ & $1(1.7)$ \\
\hline \multicolumn{3}{|l|}{ Primary diagnosis } \\
\hline Glomerulonephritis & $23(38.3)$ & $25(42.4)$ \\
\hline $\begin{array}{l}\text { Uropathy (incl. chronic } \\
\text { pyelonephritis) }\end{array}$ & $5(8.3)$ & $3(5.1)$ \\
\hline $\begin{array}{l}\text { Nephrosclerosis (incl. } \\
\text { hypertensive } \\
\text { nephropathy) }\end{array}$ & $1(1.7)$ & $3(5.1)$ \\
\hline Polycystic disease & $9(15.0)$ & 9 (15.3) \\
\hline $\begin{array}{l}\text { Other hereditary } \\
\text { nephropathy }\end{array}$ & 0 & $1(1.7)$ \\
\hline Diabetic nephropathy & 0 & $1(1.7)$ \\
\hline $\begin{array}{l}\text { Congenital } \\
\text { nephropathy }\end{array}$ & 0 & $3(5.1)$ \\
\hline Systemic vasculitis & $1(1.7)$ & 0 \\
\hline Other & $9(15.0)$ & $6(10.2)$ \\
\hline \multirow[t]{2}{*}{ Unknown } & $12(20.0)$ & $8(13.6)$ \\
\hline & $\begin{array}{l}\text { Tacrolimus } \\
\text { QD }(n=34)\end{array}$ & $\begin{array}{l}\text { Tacrolimus } \\
\text { BID }(n=32)\end{array}$ \\
\hline PK Evaluable Set & Patients (\%) & Patients (\%) \\
\hline Male & $18(52.9)$ & $26(81.3)^{3}$ \\
\hline Female & $16(47.1)$ & $6(18.8)$ \\
\hline Age $\left(\right.$ years) ${ }^{1}$ & $44.3(22-58)$ & $43.3(21-65)$ \\
\hline Height $(\mathrm{cm})^{1}$ & 167.7 (149-193) & $170.2(155-183)$ \\
\hline Weight $(\mathrm{kg})^{1}$ & $70.4(42-115)$ & $71.2(46-100)$ \\
\hline Caucasian & $33(97.1)$ & $32(100)$ \\
\hline Black & 0 & 0 \\
\hline Asian & 0 & 0 \\
\hline Other & 1 (2.9) & 0 \\
\hline $\begin{array}{l}\text { Existing glucose } \\
\text { metabolism disorder }\end{array}$ & 1 (2.9) & 0 \\
\hline
\end{tabular}

were similar for patients in both treatment groups and were maintained within the target range throughout the study (Figure 3B).

In the Full Analysis Set, all but one patient (Tacrolimus BID group) received maintenance corticosteroid therapy. Median starting doses of corticosteroids were $625 \mathrm{mg} /$ day (range: 156.3-1875 mg) and $625 \mathrm{mg} /$ day (range: 156.3$1250 \mathrm{mg}$ ) for Tacrolimus QD and Tacrolimus BID, respectively. Maintenance doses were gradually tapered to a median of $10 \mathrm{mg} /$ day (range: 5-30 mg and 10-20 mg for Tacrolimus QD and Tacrolimus BID, respectively) by 

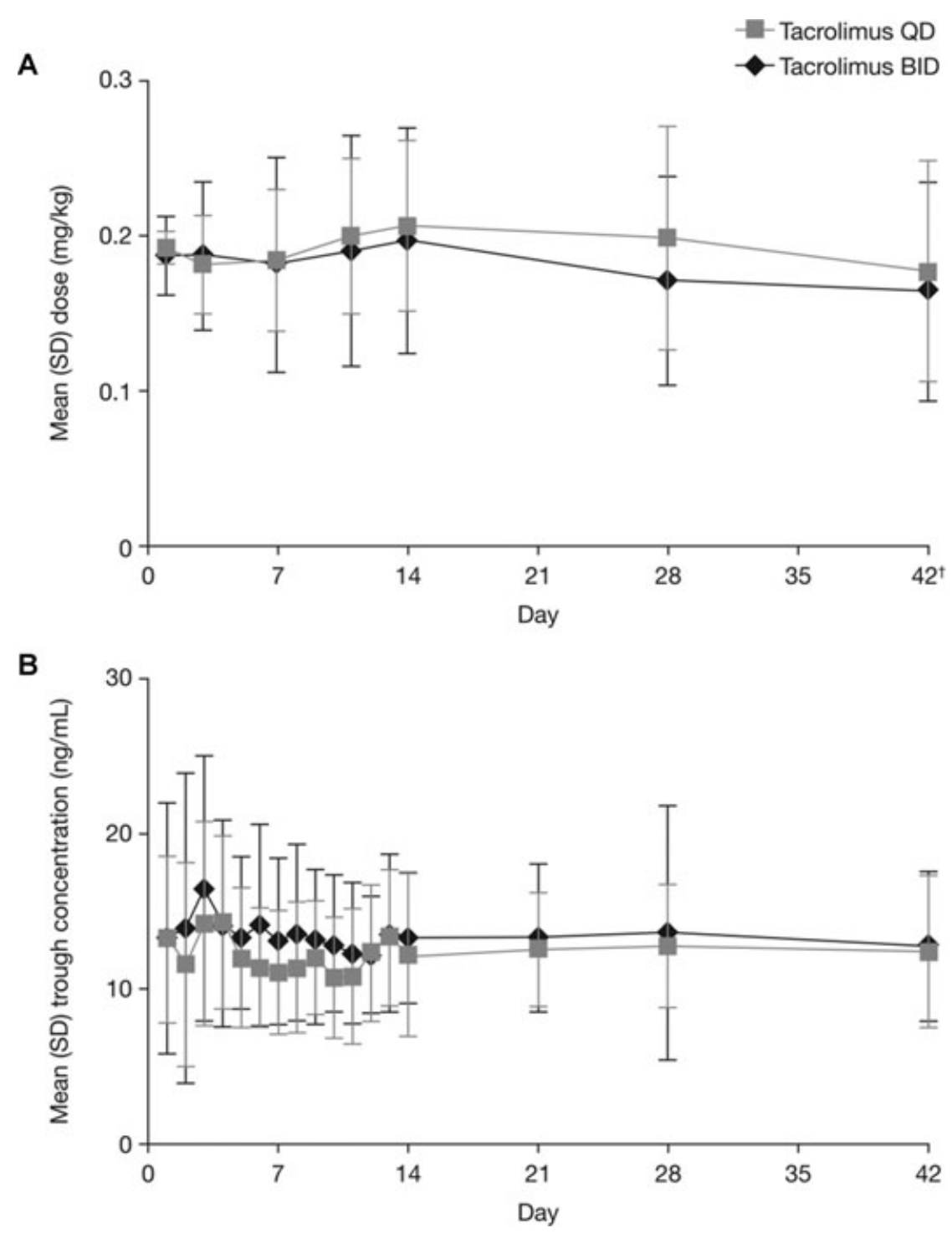

Figure 3: Summary of daily tacrolimus doses (A) and trough concentrations (B) (Full Analysis Set). ${ }^{\dagger}$ Completers only at week 6.

day 42. MMF was administered to $58 / 60(96.7 \%)$ patients receiving Tacrolimus QD, and 57/59 (96.6\%) patients receiving Tacrolimus BID. Total daily doses of MMF ranged from 0.5 to $3.0 \mathrm{~g}$ for both Tacrolimus $\mathrm{QD}$ and Tacrolimus BID treatment groups (mean dose at week 6 in completers: $1.20 \mathrm{~g} /$ day and $1.27 \mathrm{~g} /$ day, respectively).

\section{Pharmacokinetics}

On day 1 , the mean $\mathrm{AUC}_{0-24}$ was approximately $30 \%$ lower for Tacrolimus QD than for Tacrolimus BID at comparable doses (232 and $361 \mathrm{ng} . \mathrm{h} / \mathrm{mL}$, respectively; Table 2 and Figure 4). However, the mean $\mathrm{AUC}_{0-24}$ for Tacrolimus QD was comparable to Tacrolimus BID on day 14 (364 and 344 ng.h/mL, respectively) and week 6 (331 and 383 ng.h/mL, respectively). Furthermore, by day 4 , trough levels were similar for both formulations (Figure 3B). Mean $\mathrm{C}_{\text {min }}$ for Tacrolimus QD and Tacrolimus BID was within target levels at every visit.

There was a strong correlation between $\mathrm{AUC}_{0-24}$ and $\mathrm{C}_{\text {min }}$ for Tacrolimus QD and Tacrolimus BID $(r=0.83$ and $r=0.94$, respectively; Figure 5), and the slope of the line of best fit was similar for both formulations; therefore, the same therapeutic monitoring currently in place for Tacrolimus BID can also be used for Tacrolimus QD. The time to $C_{\max }\left(T_{\max }\right)$ was longer with Tacrolimus $\mathrm{QD}$ than with Tacrolimus BID, reflecting the prolonged-release characteristics of the formulation that result in extended absorption of tacrolimus compared with Tacrolimus BID (Table 2). 
Table 2: Comparison of PK parameters of tacrolimus administered as Tacrolimus QD and Tacrolimus BID (PK Evaluable Set)

\begin{tabular}{|c|c|c|}
\hline PK parameters & $\begin{array}{l}\text { Tacrolimus } \\
\mathrm{QD}(\mathrm{n}=34)\end{array}$ & $\begin{array}{c}\text { Tacrolimus } \\
\text { BID }(n=32)\end{array}$ \\
\hline Day $1^{1}$ & \multicolumn{2}{|c|}{ Mean (SD) } \\
\hline $\mathrm{AUC}_{0-24}(\mathrm{ng} \cdot \mathrm{h} / \mathrm{mL})$ & 231.91 (102.33) & 361.49 (214.65) \\
\hline $\mathrm{C}_{\max }(\mathrm{ng} / \mathrm{mL})$ & 18.24 (7.63) & 34.16 (13.86) \\
\hline $\mathrm{C}_{24}(\mathrm{ng} / \mathrm{mL})$ & $8.25(5.01)$ & 10.12 (6.98) \\
\hline $\mathrm{T}_{\max }^{2}$ (hour) & $4.4(4.3)$ & $1.7(1.0)$ \\
\hline Day $14^{3}$ & \multicolumn{2}{|c|}{ Mean (SD) } \\
\hline $\mathrm{AUC}_{0-24}(\mathrm{ng} \cdot \mathrm{h} / \mathrm{mL})$ & 363.93 (96.61) & 343.69 (105.83) \\
\hline $\mathrm{C}_{\max }(\mathrm{ng} / \mathrm{mL})$ & 29.87 (9.61) & 31.74 (12.62) \\
\hline $\mathrm{C}_{24}(\mathrm{ng} / \mathrm{mL})$ & 9.64 (3.25) & $10.02(3.04)$ \\
\hline $\mathrm{T}_{\max }$ (hour) & $2.4(1.2)$ & $1.6(0.9)$ \\
\hline Week $6^{4}$ & \multicolumn{2}{|c|}{ Mean (SD) } \\
\hline $\mathrm{AUC}_{0-24}(\mathrm{ng} \cdot \mathrm{h} / \mathrm{mL})$ & 331.49 (86.82) & $382.60(171.22)$ \\
\hline $\mathrm{C}_{\max }(\mathrm{ng} / \mathrm{mL})$ & 26.38 (7.30) & 33.04 (13.04) \\
\hline $\mathrm{C}_{24}(\mathrm{ng} / \mathrm{mL})$ & $9.60(2.93)$ & $12.06(5.91)$ \\
\hline $\mathrm{T}_{\max }$ (hour) & $2.4(1.3)$ & 1.9 (1.3) \\
\hline
\end{tabular}

${ }^{1}$ Mean daily dose on day 1 : Tacrolimus $\mathrm{QD}=0.189 \mathrm{mg} / \mathrm{kg}$; Tacrolimus BID $=0.185 \mathrm{mg} / \mathrm{kg}$.

${ }^{2}$ For Tacrolimus BID: relative to preceding morning or evening dose.

${ }^{3}$ Mean daily dose on day 14 : Tacrolimus $\mathrm{QD}=0.203 \mathrm{mg} / \mathrm{kg}$; Tacrolimus BID $=0.190 \mathrm{mg} / \mathrm{kg}$.

${ }^{4}$ Mean daily dose at week 6: Tacrolimus $\mathrm{QD}=0.175 \mathrm{mg} / \mathrm{kg}$; Tacrolimus BID $=0.164 \mathrm{mg} / \mathrm{kg}$.

\section{Efficacy over the 6-week study period}

The BPAR and survival rates were comparable between the two treatment groups during the 6-week study. The overall frequency of BPAR was $13.3 \%$ and $15.3 \%$ in the Tacrolimus QD and Tacrolimus BID groups, respectively. The Kaplan-Meier estimates for freedom from BPAR at week 6 were also similar for the two groups (Tacrolimus OD: $86.2 \%$; Tacrolimus BID: $83.1 \%)$. Graft loss was reported in one patient receiving Tacrolimus QD and three patients receiving Tacrolimus BID. The Kaplan-Meier esti-

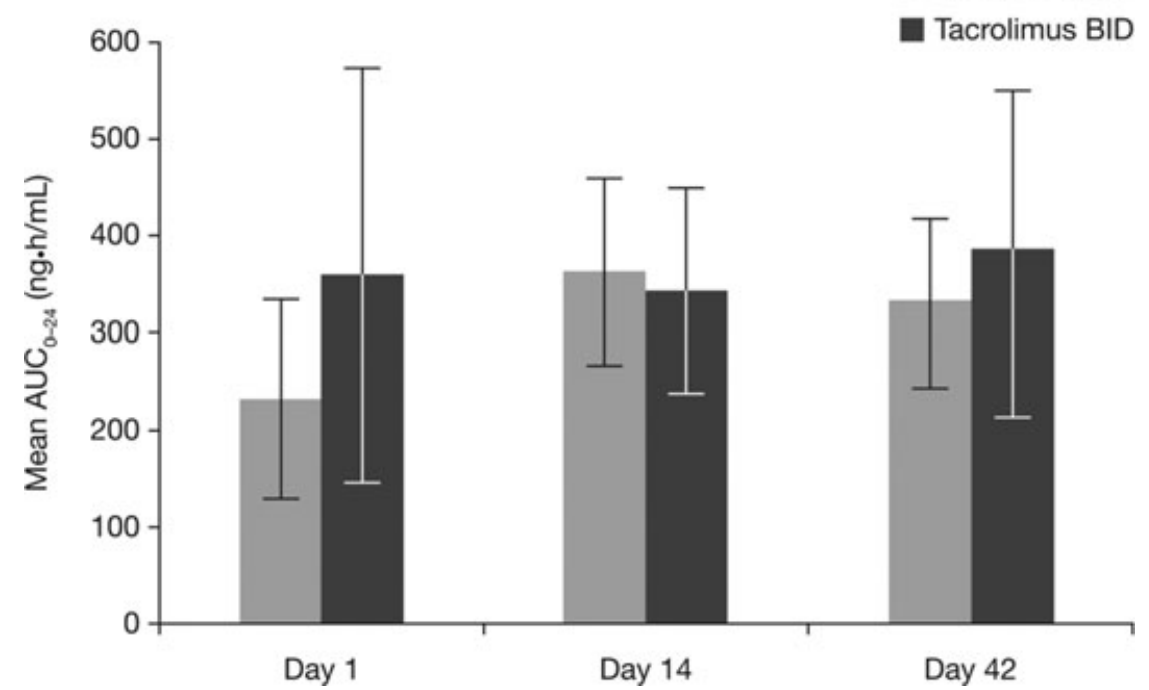

mates for graft survival for Tacrolimus QD and Tacrolimus BID were $98.3 \%$ and $93.1 \%$, respectively. There were no patient deaths during the study.

Overall, renal function was similar between the two treatment groups throughout the 6-week study period. At week 6 ; mean $( \pm S D)$ serum creatinine levels were $138.7 \pm$ 59.8 and $169.7 \pm 104.2 \mu \mathrm{mol} / \mathrm{L}(p=0.016$; Wilcoxon rank sum test), and mean $( \pm S D)$ creatinine clearance (Cockcroft-Gault) rates were $60.0 \pm 17.3$ and $54.3 \pm$ $19.5 \mathrm{~mL} / \mathrm{min}$ for Tacrolimus QD and Tacrolimus BID, respectively.

\section{Safety}

As would be expected in newly transplanted patients, the total incidence of AEs reported was high in both groups (93.3\% with Tacrolimus QD and 98.3\% Tacrolimus BID). The most frequently reported all-cause AEs in the Tacrolimus QD and Tacrolimus BID groups were: anemia (31.7\% vs. 30.5\%); nonsite specific procedural complications $(21.7 \%$ vs. $27.1 \%)$; potassium imbalance $(16.7 \%$ vs. $20.3 \%)$; and vascular hypertensive disorders not elsewhere classified (10.0\% vs. $22.0 \%$ ). There were no marked differences in the incidence, nature or severity of AEs causally related to study medication as assessed by the investigator between Tacrolimus QD and Tacrolimus BID (Figure 6). Similarly, there was no exceptional finding in any of the clinical laboratory parameters assessed.

\section{One-year follow-up}

Of 60 patients who had received at least one dose of Tacrolimus QD during the PK phase of the study, 47 $(78.3 \%)$ chose to enter the long-term extension phase (commencing at the end of the PK study, 6 weeks posttransplant). Follow-up of these 47 patients who were treated with Tacrolimus QD demonstrated safety and efficacy findings consistent with the known characteristics

\section{Tacrolimus QD}

\section{Tacrolimus BID}

Figure 4: Mean (SD) $A U C_{0-24}$ for tacrolimus. 
Figure 5: Correlation between $\mathrm{AUC}_{0-24}$ and $\mathrm{C}_{\min }$ for tacrolimus administered as Tacrolimus $Q D$ and Tacrolimus BID.
Tacrolimus QD

Tacrolimus BID of tacrolimus (6). The Kaplan-Meier estimated patient and graft survival rates after 1 year in the extension study were $100 \%$, i.e. there were no patient deaths or graft losses during this period. After 1 year of the extension study, the Kaplan-Meier estimated rate of patients free from BPAR was $93.3 \%$ and mean $( \pm S D)$ serum creatinine and creatinine clearance (Cockcroft-Gault) rates were $157.5 \pm 66.7 \mu \mathrm{mol} / \mathrm{L}$ and $57.6 \pm 19.7 \mathrm{~mL} / \mathrm{min}$, respectively.

\section{Discussion}

The primary objective of this study was to compare PK parameters of tacrolimus for Tacrolimus QD and Tacrolimus BID following kidney transplantation. When Tacrolimus
QD was initiated at the same dose as Tacrolimus BID $(0.2 \mathrm{mg} / \mathrm{kg})$, systemic exposure $\left(\mathrm{AUC}_{0-24}\right)$ on day 1 was approximately $30 \%$ lower than for Tacrolimus BID at equivalent doses. However, by day 4, trough levels were similar for both formulations. Furthermore, the observed lower exposure had no clinical consequence, as shown by similar survival rates and incidences of BPAR and AEs between the two treatment groups.

Routine therapeutic monitoring of tacrolimus includes the measurement of trough whole blood concentrations. This study demonstrated good correlation of $A \cup C_{0-24}$ to $C_{\text {min }}$ and similar slopes of the $A \cup C-C_{\text {min }}$ correlation curve for Tacrolimus QD and Tacrolimus BID in de novo kidney patients. This indicates that monitoring of whole blood trough levels provides a good estimate of systemic
Figure 6: Most frequently reported AEs assessed by the investigator as causally related to study medication (MedDRA preferred term). Full Analysis Set. No differences between Tacrolimus QD and Tacrolimus BID were significant (Fisher's exact test). NOS = not otherwise specified; MedDRA = Medical Dictionary for Regulatory Activities.

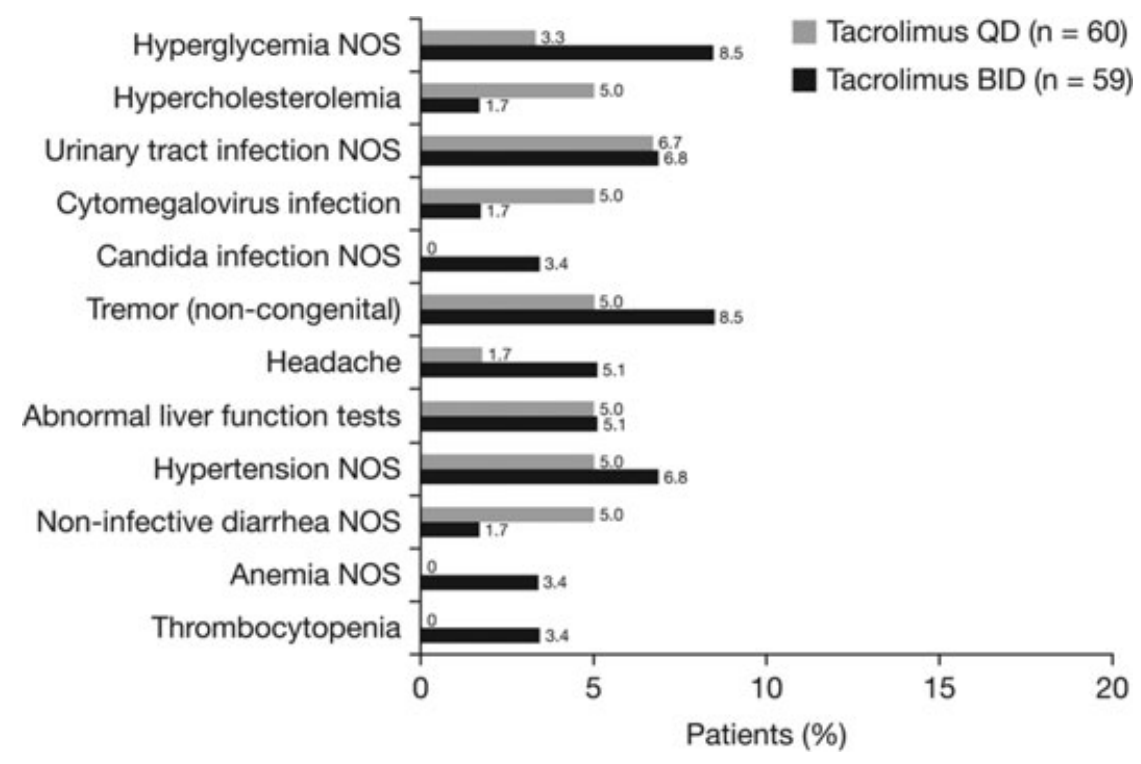


exposure and that the same trough levels could be targeted for both formulations.

Efficacy and safety profiles over the 6-week study period were similar for both formulations. The overall incidence of BPAR was comparable for Tacrolimus QD and Tacrolimus BID, with Kaplan-Meier estimates of freedom from BPAR of approximately $85 \%$ for both formulations.

Renal function was generally comparable for Tacrolimus QD and Tacrolimus BID-treated patients throughout the study. Graft loss was also comparable during the 6-week study, with excellent graft survival rates in both treatment groups, and there were no deaths.

Over the 6-week duration of the study, the safety profiles for Tacrolimus BID and Tacrolimus QD were comparable with a similar incidence and nature of AEs associated with the two formulations. There was a trend toward less hypertension and hyperglycemia in Tacrolimus QD-treated patients. Similarly, the profile of Tacrolimus QD in patients followed up for 1 year was consistent with the known safety profile of Tacrolimus BID (6). However, it should be noted that, due to the relatively low number of participants and the short duration of the study, this PK study was not designed to show differences in efficacy or safety between Tacrolimus QD and Tacrolimus BID.

This study has several strengths. First, the study was adequately powered to compare PK parameters. Second, the use of validated assay methods in determining tacrolimus concentrations ensures accurate measures of systemic exposure (12). A potential limitation of the study design is the short follow-up period (6 weeks), which is insufficient to show differences in efficacy and safety between Tacrolimus QD and Tacrolimus BID. However, 1-year follow-up of patients who were treated with Tacrolimus QD demonstrated safety and efficacy findings that are consistent with the well-documented characteristics of Tacrolimus BID. Also noteworthy is the exclusion of patients with diabetes mellitus from the study population. Two small PK studies have shown that diabetes could affect the rate and extent of tacrolimus absorption $(13,14)$. Diabetes may alter the motility of the gastric muscles, resulting in delayed gastric emptying and gastroparesis that could theoretically slow the oral absorption of drugs, including tacrolimus. In addition, a more recent PK study has also demonstrated that diabetes has a modest effect on the rate of tacrolimus absorption (15).

The results of this study in de novo kidney transplant patients showed comparable systemic steady-state tacrolimus exposure for Tacrolimus QD and Tacrolimus BID. There was a good correlation between trough tacrolimus levels and AUC for both formulations, and there was no marked difference in the efficacy and safety profiles for Tacrolimus QD and Tacrolimus BID during the 6-week study period. Thus, Tacrolimus QD could enhance post- transplant adherence and possibly improve long-term outcomes, while maintaining adequate immunosuppression.

In conclusion, Tacrolimus QD can be administered once daily in the morning on the basis of the same systemic exposure and therapeutic drug monitoring concept as Tacrolimus BID administered twice daily in the morning and evening.

\section{Acknowledgments}

The authors gratefully acknowledge the contribution from the Tacrolimus Modified Release Kidney Study Group: Z. Wlodarczyk (Poland); J.-P. Squifflet (Belgium); M. Ostrowski (Poland); E. Ancona (Italy); S. Stefoni (Italy); M. Castagneto (Italy); Y. Vanrenterghem (Belgium); B. Krämer (Germany); D. Abramowicz (Belgium); F. Oppenheimer (Spain); F. Pietruck (Germany); G. Russ (Australia); P.-A. Clavien (Switzerland); H.H. Neumayer (Germany); U. Heemann (Germany); J.J. Villafruela (Spain); D. Cantarovich (France); J. Eris (Australia); R. Walker (Australia); R. Lauzurica (Spain). The design and results of this study have been presented previously in an abstract at the Joint Annual Meeting of the American Society of Transplant Surgeons and the American Society of Transplantation, in Seattle, USA, 2005. This study and statistical analyses were supported by Astellas Pharma Europe, Staines, UK. The authors would like to thank Ify Ikelionwu, a professional medical writer with $A C U M E D{ }^{\circledR}$, for her assistance in drafting and revising the manuscript. The manuscript was compiled in compliance with the CONSORT guidelines.

Funding sources: This study and statistical analyses, and the editorial and project management services of ACUMED ${ }^{\circledR}$ in the preparation of this manuscript, were supported by Astellas Pharma Europe, Staines, UK.

ZW has participated in clinical trials sponsored by Astellas, Novartis and Roche, has received lecture fees from Astellas and has served on advisory/safety boards for Astellas and Wyeth. BK has participated in clinical trials sponsored by Astellas, BMS, Novartis, Roche and Wyeth; has received lecture fees from Astellas, Novartis and Wyeth, and has served on advisory/safety boards, and received research grants from Astellas and Novartis. FO has participated in clinical trials sponsored by, and has received consulting fees from, Novartis, Wyeth, Roche and Astellas. GR has been a consultant for Novartis, and a speaker for Wyeth and Astellas. YV has received speakers' fees from Astellas and Roche and his academic department receives research grants from Amgen, Astellas, Baxter, Janssen-Cilag and Roche. MO, FP, PR, DA, FC, JS and SS have declared no conflicts of interest. CK was an employee of Astellas at the time of the study, and NU is currently an employee of Astellas.

\section{References}

1. Gaston RS, Hudson SL, Ward M, Jones P, Macon R. Late renal allograft loss: Noncompliance masquerading as chronic rejection. Transplant Proc 1999; 31 (Suppl. 4A): 21S-23S.

2. Matas AJ, Humar A, Gillingham KJ et al. Five preventable causes of kidney graft loss in the 1990s: A single-center analysis. Kidney Int 2002; 62: 704-714.

3. Butler JA, Roderick P, Mullee M, Mason JC, Peveler RC. Frequency and impact of non-adherence to immunosuppressants after renal transplantation: A systematic review. Transplantation 2004; 77: 769-789. 


\section{Once-Daily Tacrolimus PK/De Novo Kidney Transplant}

4. Weng FL, Israni AK, Joffe MM et al. Race and electronically measured adherence to immunosuppressive medications after deceased donor renal transplantation. J Am Soc Nephrol 2005; 16 : 1839-1848.

5. Ichimaru N, Kakuta Y, Abe T et al. Treatment adherence in renal transplant recipients: A questionnaire survey on immunosuppressants. Transplant Proc 2008; 40: 1362-1365.

6. Scott L, McKeage K, Keam SJ, Plosker GL. Tacrolimus: a further update of its use in the management of organ transplantation. Drugs 2003; 63: 1247-1297.

7. Staatz CE, Tett SE. Clinical pharmacokinetics and pharmacodynamics of tacrolimus in solid organ transplantation. Clin Pharmacokinet 2004; 43: 623-653.

8. Wallemacq $\mathrm{P}$, Armstrong $\mathrm{WW}$, Brunet $\mathrm{M}$ et al. Opportunities to optimize tacrolimus therapy in solid organ transplantation: Report of the European Consensus Conference. Ther Drug Monit 2009; 31: 139-152.

9. Alloway R, Steinberg S, Khahil K et al. Conversion of stable kidney transplant recipients from a twice-daily Prograf-based regimen to a once-daily modified-release tacrolimus-based regimen. Transplant Proc 2005; 37: 867-870.

10. Florman S, Alloway R, Kalayoglu M et al. Conversion of stable liver transplant recipients from a twice-daily Prograf-based regimen to a once-daily modified release tacrolimus-based regimen. Transplant Proc 2005; 37: 1211-1213.

11. Alloway R, Vanhaecke J, Undre N, For the Modified Release Tacrolimus Heart Study Group. Conversion of stable heart transplant recipients from twice-daily Prograf to once-daily modifiedrelease tacrolimus. Transpl Int 2005; 18(Suppl 1): 75 (Abstract PO083).

12. Alak AM, Moy S, Cook M et al. An HPLC/MS/MS assay for tacrolimus in patient blood samples. Correlation with results of an ELISA assay. J Pharm Biomed Anal 1997; 16: 7-13.

13. van Duijnhoven $E$, Christiaans $M$, Schafer A, Undre N, van Hooff J. Tacrolimus dosing requirements in diabetic and nondiabetic patients calculated from pretransplantation data. Transplant Proc 1998; 30: 1266-1267.

14. van Duijnhoven E, Christiaans M, Schafer A, Undre N, Stevenson $P$, van Hooff J. The effect of breakfast on the oral bioavailability of tacrolimus in diabetic and nondiabetic patients before transplantation. Transplant Proc 1998; 30: 1268-1270.

15. Mendonza A, Zahir H, Gohh R, Akhlaghi F. Tacrolimus in diabetic kidney transplant recipients: Pharmacokinetics and application of a limited sampling strategy. Ther Drug Monit 2007; 29: 391-398. 